\title{
REVIEW
}

\section{Non-pharmacological treatment and prevention of bone loss after spinal cord injury: a systematic review}

\author{
F Biering-Sørensen ${ }^{1}$, B Hansen ${ }^{1}$ and BSB Lee ${ }^{2}$ \\ ${ }^{1}$ Clinic for Spinal Cord Injuries, Rigshospitalet, and University of Copenhagen, Copenhagen, Denmark; and ${ }^{2}$ Prince of Wales Hospital \\ Spinal Medicine Department and School of Public Health, University of New South Wales, Sydney, New South Wales, Australia
}

\begin{abstract}
Objective: Review the literature on non-pharmacological prevention and treatment of osteoporosis after spinal cord injury (SCI).

Methods: PubMed, EMBASE and the Cochrane Controlled Trials Register were searched. All identified papers were read by title, abstract and full-length article when relevant. Hand search of the articles' sources identified additional papers. For included studies, the level of evidence was determined.

Results: No studies conclusively showed an effective intervention. However, there are few randomized controlled trials (RCTs), and those that exist assess interventions and outcome measures that could be improved. Five studies on weight-bearing early post-injury are conflicting, but standing or walking may help retain bone mineral. In the chronic phase, there was no effect of weight bearing (12 studies). One study found that an early commencement of sports after $\mathrm{SCl}$ improved bone mineral, and the longer the period of athletic career, the higher the (leg) bone mineral. Early after $\mathrm{SCl}$, there may be some effects of electrical stimulation (ES) (five studies). Chronic-phase ES studies vary (14 studies, including mixed periods after injury), but improvement is seen with longer period of training, or higher frequency or stimulus intensity. Improvements correspond to trabecular bone in the distal femur or proximal tibia. Impact vibration and pulsed electromagnetic fields may have some positive effects, whereas pulsed ultrasound does not. Six studies on the influence of spasticity show inconsistent results.

Conclusions: Bone mineral should be measured around the knee; the length and intensity of the treatment should be sufficiently long and high, respectively, and should commence early after $\mathrm{SCl}$. If bone mineral is to remain, the stimulation has to be possibly continued for long term. In addition, RCTs are necessary.
\end{abstract}

Spinal Cord (2009) 47, 508-518; doi:10.1038/sc.2008.177; published online 27 January 2009

Keywords: spinal cord injury; osteoporosis; bone mineral; electrical stimulation; standing; spasticity

\section{Introduction}

Significant osteoporosis develops, in particular, in the long bones of the lower extremities in the first months and years after spinal cord injury (SCI), ${ }^{1-3}$ and may well continue years after SCI, with trabecular bone after a log curve levelling off from 1 to 3 years after SCI, whereas the cortical bone sites appear to decrease progressively beyond 10 years after SCI. ${ }^{4}$

Factors leading to more bone loss include the level of injury (tetraplegics more than paraplegics), complete lesions and longer duration after injury. Aging may also be a contributing factor. ${ }^{3-6}$

The sublesional bone loss means an increased risk of fragility fractures, in particular in the distal femur and the proximal tibia. ${ }^{7,8}$ Unfortunately, criteria for assessing fracture risk in the SCI population are lacking. ${ }^{4,9}$

Correspondence: Professor F Biering-Sørensen, Clinic for Spinal Cord Injuries, Rigshospitalet, Havnevej 25, Hornbæk DK-3100, Denmark.

E-mail: finbs@rh.regionh.dk

Received 26 September 2008; revised 8 December 2008; accepted 10 December 2008; published online 27 January 2009
In contrast to the long bones in the lower extremities, there is no obvious loss of bone in the lumbar spine, which may be because of the weight-bearing function, although degenerative changes may influence the high values found. ${ }^{3}$

There have been recent reviews on bone loss and osteoporosis in individuals after SCI. ${ }^{3,9-12}$ The present systematic review will concentrate on the evidence related to the treatment and prevention possibilities with nonpharmacological-related interventions.

\section{Methods}

For identification of articles for the review, PubMed and EMBASE were searched with no language restrictions using the following words: osteoporos*/osteopen*/bone mineral/ $\mathrm{bmc} / \mathrm{bmd}$ and spinal cord $\mathrm{inj}^{\star} /$ spinal cord lesion*/spinal cord dis*/parapleg*/tetrapleg*/quadripleg*. Searches were performed in the Cochrane Controlled Trials Register using osteoporosis/bone mineral and spinal. The PEDro database 
was hand searched under the key words: Osteoporosis and Spinal Cord Injury.

All identified papers were initially read by title, and, if relevant, by abstract. When abstracts indicated results within the purpose of the review, the full-length articles were found and reviewed.

In addition, a hand search from the other articles' sources identified additional papers that could be included.

To determine the level of evidence, the studies were judged according to the five levels used in SCIRE (Spinal Cord Injury Rehabilitation Evidence) ${ }^{13}$ (Box 1)

Two of the authors (FB-S and $\mathrm{BH}$ ) independently evaluated all studies, and those that were judged differently were discussed for possible agreement. If the first two authors were still in conflict, a third author (BL) evaluated the same paper independently to adjudicate a final decision.
Box 1 Levels of evidence - SCIRE ${ }^{13}$

Level 1 Randomized controlled trial (RCT) (PEDro score $>6$ ) Includes within-subjects comparison

Level 2 RCT (PEDro score $<6$ ); prospective controlled trial (not randomized); Cohort (prospective longitudinal study using at least two similar groups with one exposed to a particular condition)

Level 3 Case control (retrospective study comparing conditions, including historical controls)

Level 4 Pre-post (prospective trial with a baseline measure, intervention and post-test using a single group of subjects); post-test (prospective with two or more groups, intervention and then post-test using a single group of subjects); case series (retrospective study collecting variables, for example, from chart review)

Level 5 Observational (cross-sectional analysis to interpret relations); clinical consensus; case report (involving one case)

Abbreviation: SCRIE, Spinal Cord Injury Rehabilitation Evidence.

Table 1 The effect of weight bearing by standing and walking on bone in individuals with $\mathrm{SCl}$ within the first year of injury

\begin{tabular}{|c|c|c|c|c|c|c|}
\hline $\begin{array}{l}\text { Author } \\
\text { (year) }\end{array}$ & $\begin{array}{l}\text { Level of } \\
\text { evidence }\end{array}$ & Population & Design & Intervention & Measurements & Results \\
\hline $\begin{array}{l}\text { De Bruin } \\
(1999)^{14}\end{array}$ & 2 & $\begin{array}{l}13 \text { males; } 21-53 \text { years; } \\
\text { C4-T12; AIS A-D } \\
\text { Weight bearing } 1-4 \text { weeks } \\
\text { after injury }\end{array}$ & $\begin{array}{l}\text { Controlled } \\
\text { single-case, } \\
\text { experimental, } \\
\text { multiple baseline. } \\
\text { Random } \\
\text { allocation. } \\
\text { Randomization } \\
\text { method not } \\
\text { described }\end{array}$ & $\begin{array}{l}\text { Weight bearing by } \\
\text { standing and treadmill } \\
\text { walking, } 1 / 2 \rightarrow \\
\geqslant 1 \text { h day }{ }^{-1} 5 \times / \text { week } \\
\text { for half year. Monitored } \\
\text { over } 25 \text { weeks } \\
\text { (1) Immobilization: } \\
0-5 \mathrm{~h} / \text { week loading } \\
\text { exercises }(n=4) \text {; } \\
\text { (2) Standing exercises: } \\
\geqslant 5 \text { h/week }(n=5) \text {; } \\
(3) \text { Combined standing } \\
\text { and walking exercises: } \\
\geqslant 5 \mathrm{~h} / \text { week }(n=4)\end{array}$ & $\begin{array}{l}\text { BMD by } \mathrm{pQCT} \text { of the } \\
\text { tibia } 5 \text { and } 25 \text { weeks } \\
\text { after injury } \\
\text { Flexural wave } \\
\text { propagation velocity } \\
\text { with a biomechanical } \\
\text { testing method }\end{array}$ & $\begin{array}{l}\text { Decrease in } \\
\text { trabecular bone with } \\
\text { non-intervention, } \\
\text { early mobilization } \\
\text { gave no or } \\
\text { insignificant loss of } \\
\text { trabecular bone. No } \\
\text { difference between } \\
\text { Groups } 2 \text { and } 3\end{array}$ \\
\hline $\begin{array}{l}\text { Frey-Rindova } \\
(2000)^{15}\end{array}$ & 4 & $\begin{array}{l}24 \text { males; } 19-59 \text { years; } \\
\text { traumatic SCI C4-L1; } \\
\text { Frankel A-C } \\
\text { Weight bearing } 1-4 \text { weeks } \\
\text { after injury }\end{array}$ & $\begin{array}{l}\text { Prospective } \\
\text { repeated } \\
\text { measure }\end{array}$ & $\begin{array}{l}\text { Standing/walking } \\
\text { at least } 30 \text { min } \\
3 \text { times/week } \\
\text { No intervention } \\
\text { (because of motivational } \\
\text { or health problems): } \\
8 . \text { Intervention: } 16\end{array}$ & $\begin{array}{l}\text { BMD by } \mathrm{pQCT} \text { of the } \\
\text { tibia } 1,6,12 \text { months } \\
\text { after } \mathrm{SCl}\end{array}$ & $\begin{array}{l}\text { Standing/walking } \\
\text { program did not } \\
\text { significantly influence } \\
\text { the BMD loss }\end{array}$ \\
\hline Ben $(2005)^{16}$ & 1 & $\begin{array}{l}20 \text { (4 females); } 34 \text { years } \\
\text { (s.d. }=15) ; 4 \text { months } \\
\text { (s.d. }=2 \text { ) after injury; } \\
<2 / 5 \text { strength in the } \\
\text { lower limbs ( } 100 \text { screened) }\end{array}$ & $\begin{array}{l}\text { Assessor blinded } \\
\text { within-subject } \\
\text { contralateral limb } \\
\text { randomized } \\
\text { controlled trial }\end{array}$ & $\begin{array}{l}40 \text { legs } \\
\text { Weight bearing } \\
\text { through one leg on a } \\
\text { tilt table for } 30 \text { min } \\
3 \times / \text { week for } 12 \text { weeks }\end{array}$ & $\begin{array}{l}\text { BMD by DXA of the } \\
\text { proximal femur }\end{array}$ & $\begin{array}{l}\text { No effect on femoral } \\
\text { BMD }\end{array}$ \\
\hline $\begin{array}{l}\text { Giangregorio } \\
(2005)^{17}\end{array}$ & 4 & $\begin{array}{l}5 \text { traumatic } C 3-C 8 \text {, } \\
\text { AIS B-C, within } 2-6 \text { months } \\
\text { of injury }\end{array}$ & $\begin{array}{l}\text { Prospective } \\
\text { case series }\end{array}$ & $\begin{array}{l}48 \text { sessions: twice } \\
\text { weekly BWSTT for } \\
6-8 \text { months }\end{array}$ & $\begin{array}{l}\text { BMD by DXA of the } \\
\text { lumbar spine, proximal } \\
\text { femora, proximal tibia }\end{array}$ & $\begin{array}{l}\text { BWSTT did not } \\
\text { prevent bone loss }\end{array}$ \\
\hline $\begin{array}{l}\text { Alekna } \\
(2008)^{18}\end{array}$ & 2 & $\begin{array}{l}54 \text { (10 females, } 44 \text { males) } \\
\text { traumatic AIS A-B; C2-L1; } \\
21 \text { tetraplegic, } 33 \\
\text { paraplegic; female age } \\
37.3 \pm 10.0 \text { years; male age } \\
33.4 \pm 12.0 \text { years }\end{array}$ & $\begin{array}{l}\text { Prospective } \\
\text { matched pairs }\end{array}$ & $\begin{array}{l}27 \text { gender, age and } \\
\text { height matched in } \\
\text { each group: } \\
\text { Standing group } \\
\text { (standing } \geqslant 1 \mathrm{~h} / \text { day } \\
\geqslant 5 \text { days/week) } \\
\text { Non-standing (not } \\
\text { standing at all) }\end{array}$ & $\begin{array}{l}\text { BMD by DXA of arms, } \\
\text { legs, pelvis and L2-L4. } \\
6-16 \text { weeks, } 12 \text { and } \\
24 \text { months after injury }\end{array}$ & $\begin{array}{l}\text { Standing group: } \\
\text { significant higher } \\
\text { BMD in legs } \\
(P=0.0004) \text { and } \\
\text { pelvis }(P=0.014) \\
\text { after } 2 \text { years }\end{array}$ \\
\hline
\end{tabular}

Abbreviations: AIS, American Spinal Injury Association (ASIA) Impairment Scale; BMD, bone mineral density; BWSTT, body weight-supported treadmill training; DXA, dual-energy X-ray absorptiometry; $\mathrm{pQCT}$, peripheral quantitative computed tomography; $\mathrm{SCl}$, spinal cord injury. 
Table 2 The effect of weight bearing by standing and walking on bone in individuals with $\mathrm{SCl}$ more than a year after injury

\begin{tabular}{|c|c|c|c|c|c|c|}
\hline $\begin{array}{l}\text { Author } \\
\text { (year) }\end{array}$ & $\begin{array}{l}\text { Level of } \\
\text { evidence }\end{array}$ & Population & Design & Intervention & Measurements & Results \\
\hline $\begin{array}{l}\text { Biering- } \\
\text { Sørensen } \\
(1988)^{19}\end{array}$ & 5 & $\begin{array}{l}16 \text { with paralegia; } 16 \text { years } \\
\text { and older at } \mathrm{SCl} ; 2-25 \\
\text { years after } \mathrm{SCl}\end{array}$ & $\begin{array}{l}\text { Cross-sectional } \\
\text { Descriptive }\end{array}$ & $\begin{array}{l}\text { Long leg braces: } \\
\text { minimum } 1 \mathrm{~h} \text { daily: } 5 \text {; } \\
\text { Not using long leg } \\
\text { braces: } 11\end{array}$ & $\begin{array}{l}\text { BMC by DPA of the } \\
\text { lumbar spine, femoral } \\
\text { neck and shaft, and } \\
\text { proximal tibia }\end{array}$ & $\begin{array}{l}\text { No effect of the use of } \\
\text { long leg braces }\end{array}$ \\
\hline $\begin{array}{l}\text { Saltzstein } \\
(1992)^{20}\end{array}$ & 5 & $\begin{array}{l}20 \text { complete } \mathrm{SCl} ; \sim 20 \\
\text { years after injury; } 7 \\
\text { incomplete } \mathrm{SCl} ; \sim 10 \text { years } \\
\text { after injury; } 15 \text { able-bodied } \\
\text { controls. All males; age } \\
42 \pm 13 \text { years }\end{array}$ & $\begin{array}{l}\text { Cross-sectional } \\
\text { Descriptive }\end{array}$ & $\begin{array}{l}\text { Mobility index from } 1 . \\
\text { Complete paralysis to } 9 \text {. } \\
\text { Able bodied-unlimited } \\
\text { ambulation: } \\
\text { (1) } 7 \text { incomplete } \mathrm{SCl} \text {; } \\
\text { (2) } 20 \text { complete } \mathrm{SCl} \text {; } \\
\text { and } \\
\text { (3) } 15 \text { able-bodied } \\
\text { controls }\end{array}$ & $\begin{array}{l}\text { BMD by single-photon } \\
\text { absorptiometry of } \\
\text { distal tibia }\end{array}$ & $\begin{array}{l}\text { BMD was positively } \\
\text { correlated with } \\
\text { mobility index scores } \\
(P<0.01) \text {. Difficult to } \\
\left.\text { understand (Figure } 2^{20}\right) \\
\text { and the correlation, as } \\
20 \text { should be mobility } \\
\text { index } 1 \text {, and } 15 \text { index } \\
\text { score } 9 \text { ? }\end{array}$ \\
\hline $\begin{array}{l}\text { Kunkel } \\
(1993)^{21}\end{array}$ & 4 & $\begin{array}{l}6 \text { C5-T12 males; } 36-65 \\
\text { years; } 4 \text { SCI for } 10-28 \\
\text { years, } 2 \text { multiple sclerosis }\end{array}$ & Pre-post trial & $\begin{array}{l}\text { Standing frame: } \\
10 \rightarrow 30 \text { min } 3 \times / \text { day, } \\
\text { changed to } 45 \text { min } \\
2 \times / \text { day for } 5 \text { months }\end{array}$ & $\begin{array}{l}\text { BMD by DPA of the } \\
\text { lumbar spine and } \\
\text { femoral neck }\end{array}$ & No effect \\
\hline $\begin{array}{l}\text { Ogilvie } \\
(1993)^{22}\end{array}$ & 4 & $\begin{array}{l}4 \text { ( } 2 \text { females) paraplegic, } \\
16-42 \text { years. Wheelchair } \\
\text { for at least } 1 \text { year }\end{array}$ & Pre-post trial & $\begin{array}{l}\text { Reciprocating gait } \\
\text { orthosis for } 24-30 \\
\text { months }\end{array}$ & $\begin{array}{l}\text { BMD by a quantitative } \\
\text { CT of the lumbar spine } \\
\text { and femoral neck }\end{array}$ & $\begin{array}{l}3 \text { of } 4 \text { increased or } \\
\text { maintained femoral } \\
\text { neck BMD }\end{array}$ \\
\hline $\begin{array}{l}\text { Goemaere } \\
(1994)^{23}\end{array}$ & 5 & $\begin{array}{l}53 \text { with complete } \\
\text { traumatic paraplegia for } \\
\text { at least } 1 \text { year }\end{array}$ & $\begin{array}{l}\text { Cross-sectional } \\
\text { Descriptive }\end{array}$ & $\begin{array}{l}\text { None-15 did not and } \\
38 \text { did regularly perform } \\
\text { passive weight bearing: } \\
\text { long leg braces: } 20 ; \\
\text { standing frame: } 9 ; \\
\text { standing wheelchair: } 9\end{array}$ & $\begin{array}{l}\text { BMD by DXA of the } \\
\text { lumbar spine, hip and } \\
\text { the femoral shaft }\end{array}$ & $\begin{array}{l}\text { Femoral shaft higher } \\
\text { BMD }(P=0.009) \text { in } \\
\text { the standing group }\end{array}$ \\
\hline $\begin{array}{l}\text { Thoumie } \\
(1995)^{24}\end{array}$ & 4 & $\begin{array}{l}7 \text { (1 female) T2-T10; } \\
26-33 \text { years; SCI 15-60 } \\
\text { months }\end{array}$ & Pre-post trial & $\begin{array}{l}\text { Gait rehabilitation } \\
\text { program with } \\
\text { reciprocating gait } \\
\text { hybrid orthoses } \\
\text { (RGO-II). Training } \\
\text { program with } 2 \mathrm{~h} \\
\text { sessions } 3 \times / \text { week } \\
\text { (14-18 months) }\end{array}$ & $\begin{array}{l}\text { BMD by DPA of the } \\
\text { lumbar spine and } \\
\text { femoral neck }\end{array}$ & No gain in BMD \\
\hline $\begin{array}{l}\text { Needham- } \\
\text { Shropshire } \\
(1997)^{25}\end{array}$ & 4 & $\begin{array}{l}16 \text { ( } 3 \text { females), T4-T11 } \\
\text { complete SCI. Mean age } \\
28.8 \text { years, mean } \\
3.8 \text { years after injury }\end{array}$ & Pre-post trial & $\begin{array}{l}32 \text { sessions, } \\
\text { approximately } \\
12 \text { weeks, using } \\
\text { Parastep1 ambulation } \\
\text { system }\end{array}$ & $\begin{array}{l}\text { BMD by DPA of the } \\
\text { femoral neck, head and } \\
\text { Ward's triangle }\end{array}$ & $\begin{array}{l}\text { No change in BMD } \\
\text { using repeated } \\
\text { measures analyses } \\
\text { of variance }\end{array}$ \\
\hline $\begin{array}{l}\text { Frey-Rindova } \\
(2000)^{15}\end{array}$ & 4 & $\begin{array}{l}24 \text { males, traumatic C4-L1, } \\
\text { Frankel A-C; 19-59 years, } \\
\text { weight bearing 1-4 weeks } \\
\text { after injury }\end{array}$ & $\begin{array}{l}\text { Prospective } \\
\text { repeated measure }\end{array}$ & $\begin{array}{l}\text { Standing/walking } \\
\text { at least } 30 \text { min } 3 \text { times/ } \\
\text { week: No (due to } \\
\text { motivational or health } \\
\text { problems): } 8 \text {, Yes: } 16\end{array}$ & $\begin{array}{l}\text { BMD by } \mathrm{pQCT} \text { of tibia } \\
\text { for } 1,6,12 \text { months } \\
\text { after } \mathrm{SCl}\end{array}$ & $\begin{array}{l}\text { Standing/walking did } \\
\text { not significantly } \\
\text { influence the } \\
\text { BMD loss }\end{array}$ \\
\hline $\begin{array}{l}\text { Dauty } \\
(2000)^{26}\end{array}$ & 5 & $\begin{array}{l}31 \text { males; } 22 \text { AIS A, } 4 \text { AIS B, } \\
3 \text { AIS C, } 2 \text { AIS D; } 36 \pm 12.3 \\
\text { years (range 18-60); more } \\
\text { than } 1 \text { year after injury }\end{array}$ & $\begin{array}{l}\text { Cross-sectional } \\
\text { Descriptive }\end{array}$ & $\begin{array}{l}\text { Duration of daily } \\
\text { verticalization: } \\
\text { (1) }<1 \mathrm{~h} / \text { day; } \\
\text { (2) } 1 \mathrm{~h} / \text { day; and } \\
\text { (3) }>1 \mathrm{~h} / \text { day } \\
\text { Long leg braces used } \\
\text { by six individuals }\end{array}$ & $\begin{array}{l}\text { BMD/BMC by DPA the } \\
\text { of lumbar spine, } \\
\text { femoral neck, distal } \\
\text { femur and proximal } \\
\text { tibia }\end{array}$ & $\begin{array}{l}\text { No significant } \\
\text { difference between } \\
\text { groups } \\
\text { No correlation } \\
\text { between use of long } \\
\text { leg braces and BMC } \\
\text { of the lower limb }\end{array}$ \\
\hline Sabo $(2001)^{27}$ & 5 & $\begin{array}{l}46 \text { C } 4-T 12 \text { males; } 24 \\
\text { cervical, } 22 \text { thoracic } \\
\text { lesions; } 33 \text { Frankel } A, 13 \\
\text { Frankel B, C, D; } 32.0 \pm \\
10.7 \text { years (mean } \pm \text { s.e.m.); } \\
8 \text { years after injury } \\
\text { (1-26 years) }\end{array}$ & $\begin{array}{l}\text { Cross-sectional } \\
\text { Descriptive }\end{array}$ & $\begin{array}{l}7 \text { in ambulatory group, } \\
\text { that is, household or } \\
\text { community walker } \\
39 \text { non-ambulatory } \\
\text { group, that is, wheelchair } \\
\text { or therapeutic standing } \\
\text { only }\end{array}$ & $\begin{array}{l}\text { BMD by DXA of the } \\
\text { proximal femur, } \\
\text { lumbar spine and } \\
\text { distal forearm }\end{array}$ & $\begin{array}{l}\text { Ambulatory status } \\
\text { had no significant } \\
\text { influence on BMD }\end{array}$ \\
\hline
\end{tabular}


Table 2 Continued

\begin{tabular}{|c|c|c|c|c|c|c|}
\hline $\begin{array}{l}\text { Author } \\
\text { (year) }\end{array}$ & $\begin{array}{l}\text { Level of } \\
\text { evidence }\end{array}$ & Population & Design & Intervention & Measurements & Results \\
\hline Eser $(2005)^{28}$ & 5 & $\begin{array}{l}54 \text { ( } 5 \text { females) C5-L3 AIS A } \\
\text { and B; } 24-72 \text { years; } 5-48 \\
\text { years after injury; } 6 \text { acquired } \\
\text { SCI before the age of } 18 \\
\text { years }\end{array}$ & $\begin{array}{l}\text { Cross-sectional } \\
\text { Descriptive }\end{array}$ & $\begin{array}{l}\text { Divided into: (1) no } \\
\text { standing; } \\
\text { (2) passive standing } \\
\leqslant 2 \mathrm{~h} / \text { week; } \\
\text { (3) passive standing } \\
>2 \mathrm{~h} / \text { week; and } \\
\text { (4) passive standing } \\
>1 \mathrm{~h} / \text { day }\end{array}$ & $\begin{array}{l}\mathrm{BMD} \text { by } \mathrm{PQCT} \text { of the } \\
\text { radius, femur and tibia }\end{array}$ & $\begin{array}{l}\text { No relation to bone } \\
\text { status }\end{array}$ \\
\hline $\begin{array}{l}\text { Giangregorio } \\
(2006)^{11}\end{array}$ & 4 & $\begin{array}{l}13 \text { ( } 2 \text { females) C } 4-T 12 \\
\text { AIS B-C; age } 20-53 \text { years; } \\
1.2-24 \text { years after injury }\end{array}$ & Pre-post trial & $\begin{array}{l}12 \text { months BWSTT: } 3 \\
\text { times/week for } 144 \\
\text { sessions }\end{array}$ & $\begin{array}{l}\text { BMD by DXA of the } \\
\text { spine, proximal and } \\
\text { distal femur, and } \\
\text { proximal tibia }\end{array}$ & $\begin{array}{l}\text { No significant } \\
\text { changes in BMD or } \\
\text { bone geometry at } \\
\text { any site. Whole-body } \\
\text { BMD decreased }\end{array}$ \\
\hline $\begin{array}{l}\text { Goktepe } \\
(2008)^{29}\end{array}$ & 5 & $\begin{array}{l}71 \text { ( } 11 \text { females); } 64 \\
\text { traumatic, } 7 \text { non-traumatic; } \\
56 \text { paraplegia, } 15 \\
\text { tetraplegia, AIS A-B. } \\
18-46 \text { years; } \\
\text { at least } 1 \text { year after injury }\end{array}$ & Cross-sectional & $\begin{array}{l}\text { (1) Standing } \geqslant 1 \mathrm{~h} / \text { day } \\
\text { (2) Standing }<1 \mathrm{~h} / \text { day } \\
\text { (3) Non-standing }\end{array}$ & $\begin{array}{l}\text { BMD by DXA of L2-L4, } \\
\text { hips, trochanters, } \\
\text { Wards's triangles and } \\
\text { femoral necks }\end{array}$ & $\begin{array}{l}\text { No difference } \\
\text { between groups on } \\
\text { any site }\end{array}$ \\
\hline
\end{tabular}

Abbreviations: AIS, American Spinal Injury Association (ASIA) Impairment Scale; BMC, bone mineral content; BMD, bone mineral density; BWSTT, body weightsupported treadmill training; CT, computer tomography; DPA, dual-energy photon absorptiometry; DXA, dual-energy X-ray absorptiometry; pQCT, peripheral quantitative computed tomography; $\mathrm{SCl}$, spinal cord injury.

Table 3 The effect of exercise on bone in individuals with SCI

\begin{tabular}{|c|c|c|c|c|c|c|}
\hline $\begin{array}{l}\text { Author } \\
\text { (year) }\end{array}$ & $\begin{array}{l}\text { Level of } \\
\text { evidence }\end{array}$ & Population & Design & Intervention & Measurements & Results \\
\hline $\begin{array}{l}\text { Jones } \\
(2002)^{30}\end{array}$ & 5 & $\begin{array}{l}17 \text { C4-L3 Frankel A-C } \\
\text { males, } 7-372 \text { months } \\
\text { after injury, } 32 \pm 8 \\
\text { years and } 17, \\
\text { weight- and height- } \\
\text { matched able-bodied } \\
\text { males }\end{array}$ & $\begin{array}{l}\text { Cross-sectional } \\
\text { descriptive } \\
\text { comparing active } \\
\text { with SCI and } \\
\text { able-bodied }\end{array}$ & $\begin{array}{l}\text { SCI males: } \\
442 \pm 225 \text { min/week } \\
\text { physical activity } \\
\text { Able-bodied: } \\
367 \pm 167 \text { min/week } \\
\text { physical activity }\end{array}$ & $\begin{array}{l}\text { BMD, BMC by DXA } \\
\text { of total body, } \\
\text { hip and leg }\end{array}$ & $\begin{array}{l}\mathrm{BMD} / \mathrm{BMC} \text { lower in the } \mathrm{SCl} \\
\text { group }(P=0.0001) . \\
\text { Lumbar and arm no } \\
\text { difference }\end{array}$ \\
\hline $\begin{array}{l}\text { Goktepe } \\
(2004)^{31}\end{array}$ & 5 & $\begin{array}{l}34 \text { AIS A-C males mean } \\
\text { age } 28.5 \text { years, } 6.2 \\
\text { years after injury }\end{array}$ & $\begin{array}{l}\text { Cross-sectional } \\
\text { descriptive } \\
\text { comparing elite } \\
\text { basketball players } \\
\text { and sedentary } \\
\text { persons with } \mathrm{SCl}\end{array}$ & $\begin{array}{l}17 \text { paraplegic basketball } \\
\text { players } \\
17 \text { sedentary paraplegic } \\
\text { persons }\end{array}$ & $\begin{array}{l}\text { BMD by DXA of the } \\
\text { distal radius of the } \\
\text { dominant arm, lumbar, } \\
\text { trochanters, Ward's } \\
\text { triangle and femoral } \\
\text { neck }\end{array}$ & $\begin{array}{l}\text { No difference between } \\
\text { groups except distal radial } \\
\text { BMD higher in basketball } \\
\text { players }(P=0.01)\end{array}$ \\
\hline $\begin{array}{l}\text { Miyahara } \\
(2008)^{32}\end{array}$ & 5 & $\begin{array}{l}28 \text { paraplegic; } \\
34.7 \pm 9.3 \\
\text { years; } 14.6 \pm 8.4 \\
\text { years after injury }\end{array}$ & $\begin{array}{l}\text { Cross-sectional } \\
\text { descriptive }\end{array}$ & Wheelchair athletes & $\begin{array}{l}\text { BMD by DXA of the } \\
\text { entire body, both } \\
\text { arms and legs, and } \\
\text { body trunk }\end{array}$ & $\begin{array}{l}\text { The earlier the athlete } \\
\text { restarted sports after injury, } \\
\text { the higher the BMD of legs } \\
(P=0.019), \text { body } \\
\text { trunk }(P=0.018) \text { and the } \\
\text { entire body }(P=0.011) \text {. } \\
\text { The period of athletic } \\
\text { career after restating was } \\
\text { significantly related with } \\
\text { leg BMD }(P=0.022)\end{array}$ \\
\hline
\end{tabular}

Abbreviations: AIS, American Spinal Injury Association (ASIA) Impairment Scale; BMC, bone mineral content; BMD, bone mineral density; DXA, dual-energy X-ray absorptiometry; $\mathrm{SCl}$, spinal cord injury.

\section{Results and discussion}

The Pedro Search identified nine articles for individual review (including review articles for bibliographic searching), whereas the EMBASE review revealed 415 references and the Medline revealed 349 references for abstract review. A total of 45 studies were explored in detail (Tables 1-7). Seventeen studies analyzed the effect of weight 
Table 4 The effect of electrical stimulation on bone in individuals with $\mathrm{SCl}$ early after injury

\begin{tabular}{|c|c|c|c|c|c|c|}
\hline $\begin{array}{l}\text { Author } \\
\text { (year) }\end{array}$ & $\begin{array}{l}\text { Level of } \\
\text { evidence }\end{array}$ & Population & Design & Intervention & Measurements & Results \\
\hline Eser $(2003)^{33}$ & 2 & $\begin{array}{l}38-19 \text { in each group } \\
\text { comparable regarding age, } \\
\text { and lesion level: C5-T12, } \\
\text { AIS A-B; } 2 \text { females in each } \\
\text { group; } 20-60 \text { years; } 1-3 \\
\text { months after injury }\end{array}$ & $\begin{array}{l}\text { Self-selection for } \\
\text { intervention or } \\
\text { control } \\
\text { Pre-Post trial }\end{array}$ & $\begin{array}{l}\text { Intervention: Up to } \\
30 \text { min FES cycling } 3 \\
\text { days/week; }+2 \text { days/ } \\
\text { week } 30 \text { min passive } \\
\text { standing } \\
\text { Control: } 5 \text { days/week } \\
30 \text { min passive standing } \\
\text { For the period of their } \\
\text { primary rehabilitation } \\
\text { (mean } 6 \text { months) }\end{array}$ & $\begin{array}{l}\text { BMD by } C T \text { of the right } \\
\text { tibia diaphysis }\end{array}$ & No significant effect \\
\hline $\begin{array}{l}\text { Shields } \\
(2006)^{34}\end{array}$ & 2 & $\begin{array}{l}7 \text { C5-T10 AIS A males; } \\
21-43 \text { years; within first } \\
6 \text { weeks of injury }\end{array}$ & $\begin{array}{l}\text { Within-subject, } \\
\text { contralateral limb } \\
\text { control } \\
\text { Pre-Post trial }\end{array}$ & $\begin{array}{l}\text { Training } 1.9-3.1 \text { years } \\
\text { with } 83 \% \text { compliance: } \\
\text { unilateral tibial nerve } \\
\text { electrical stimulation for } \\
\text { plantar flexion. } 4 \text { bouts } \\
\text { of } 125 \text { contractions/day, } \\
5 \text { days/week }\end{array}$ & $\begin{array}{l}\text { BMD by pQCT of the } \\
\text { distal tibia (only } 4 \text { males) }\end{array}$ & $\begin{array}{l}\text { BMD was higher in } \\
\text { the trained limb than } \\
\text { in the untrained limb } \\
(P<0.05)\end{array}$ \\
\hline $\begin{array}{l}\text { Shields } \\
(2006)^{35}\end{array}$ & 2 & $\begin{array}{l}6 \mathrm{C} 5-\mathrm{T} 10 \text { AIS A; age at SCI } \\
21-43 \text { years; } 0.16-0.35 \\
\text { years after injury }\end{array}$ & $\begin{array}{l}\text { Repeated } \\
\text { measures, within- } \\
\text { subject } \\
\text { contralateral limb } \\
\text { control }\end{array}$ & $\begin{array}{l}\text { Training } 1.7-3 \text { years with } \\
\text { compliance } 70.9-93.9 \% \text { : } \\
\text { Unilateral electrical } \\
\text { stimulation of plantar } \\
\text { flexor muscles. } 4 \text { bouts of } \\
120 \text { contractions/day, } 5 \\
\text { days/week }\end{array}$ & $\begin{array}{l}\text { BMD by DXA of the } \\
\text { spine, hips and knees }\end{array}$ & $\begin{array}{l}\text { Decline in tibia } B M D \\
\text { in the trained less } \\
\text { than in untrained } \\
(P<0.05)\end{array}$ \\
\hline Clark $(2007)^{36}$ & 2 & $\begin{array}{l}33 \text { AIS A-D: FES group } 23 \\
\text { C4-Th10; age } 29 \pm 9 \text { years; } \\
10 \text { lost for } 6 \text { months follow- } \\
\text { up. Controls } 10 \text { C } 5 \text {-Th } 12 \text {; } \\
31 \pm 11 \text { years; } 3 \text { lost for } 6 \\
\text { months follow-up }\end{array}$ & $\begin{array}{l}\text { Controlled repeat } \\
\text { measures study }\end{array}$ & $\begin{array}{l}\text { Two weeks } \\
\text { preconditioning. FES to } \\
\text { quadriceps femoris and } \\
\text { anterior tibial muscles } \\
2 \times 15 \text { min FES/leg, } 5 \\
\text { days/week for } 5 \text { months }\end{array}$ & $\begin{array}{l}\text { BMD by DXA of the total } \\
\text { body, spine and hip } \\
\text { within } 3 \text { weeks, } 6 \text { weeks } \\
3 \text { and } 6 \text { months after } \\
\text { injury }\end{array}$ & $\begin{array}{l}\text { Three months after } \\
\text { injury, the FES group } \\
\text { had higher total body } \\
\text { BMD }(P<0.01) \text {, but } \\
\text { not thereafter and } \\
\text { not for the hip or } \\
\text { spine }\end{array}$ \\
\hline $\begin{array}{l}\text { Dudley- } \\
\text { Javoroski } \\
(2008)^{37}\end{array}$ & 5 & $\begin{array}{l}\text { One T4 AIS: A male } 21 \\
\text { years, } 7 \text { weeks after injury }\end{array}$ & $\begin{array}{l}\text { Single-case } \\
\text { prospective, } \\
\text { within-subject, } \\
\text { contralateral limb } \\
\text { control } \\
\text { (randomized) }\end{array}$ & $\begin{array}{l}\text { Unilateral soleus muscle } \\
\text { electrical stimulation over } \\
4.8 \text { years home-based } \\
\text { training } 30 \mathrm{~min} / \text { day, } 5 \\
\text { days/week }\end{array}$ & $\begin{array}{l}\text { BMD by pQCT of the } \\
\text { distal tibia every } 6 \\
\text { months }\end{array}$ & $\begin{array}{l}\text { BMD of the untrained } \\
\text { tibia declined } \sim 14 \% \\
\text { per year, and } 7 \% \text { in } \\
\text { the trained tibia. } \\
\text { BMD preferentially } \\
\text { preserved in the } \\
\text { posterior half of the } \\
\text { tibia with annual } \\
\text { decline of } 2.6 \%\end{array}$ \\
\hline
\end{tabular}

Abbreviations: AIS, American Spinal Injury Association (ASIA) Impairment Scale; BMC, bone mineral content; BMD, bone mineral density; C, complete spinal cord lesion; DXA, dual-energy X-ray absorptiometry; FES, functional electrical stimulation; IC, incomplete spinal cord lesion; pQCT, peripheral computed tomography; $\mathrm{SCl}$, spinal cord injury.

bearing by standing and walking, of which five included data from the first year after injury. Three studies looked at the effect of exercise, 19 studies analyzed electrical stimulation, 3 looked at other physical interventions and 6 studies made some reference to the effect of spasticity/spasms. Two studies only showed adequate randomization, allocation concealment and blinding (PEDro score $\geqslant 6$ ). ${ }^{16,54}$ Both studies showed no effect, used a within-subjects study design and dual-energy X-ray absorptiometry but analyzed different outcome measures. Ben et al. ${ }^{16}$ used bone mineral density (BMD) at the proximal femur and Warden et al. ${ }^{54}$ used bone mineral content (BMC) at the heel. The intervention group for Ben et al. ${ }^{16}$ (Table 1) was standing weight bearing on one leg on a tilt table for $30 \mathrm{~min}$, thrice weekly for 12 weeks, whereas Warden et al. ${ }^{54}$ (Table 6) carried out a low-intensity pulsed ultrasound on one heel for $20 \mathrm{~min} \mathrm{day}^{-1}, 5$ days/week over 6 weeks. None of these considered bone changes around the knee, where the majority of bone loss occurs after SCI.

The effect of weight bearing by standing and walking The studies on weight bearing from the early period (within the first year) after SCI (Table 1) are conflicting. The single level 1 study by Ben et al. ${ }^{16}$ showed that early-period weight bearing did not increase BMD; however, the therapy period was relatively short and only involved simple static weight bearing compared with the other two studies showing positive results within this group. In addition, BMD was 
Table 5 The effect of electrical stimulation on bone in individuals with SCI longer time after injury or including mixed periods after injury

\begin{tabular}{|c|c|c|c|c|c|c|}
\hline Author (year) & $\begin{array}{l}\text { Level of } \\
\text { evidence }\end{array}$ & Population & Design & Intervention & Measurements & Results \\
\hline Pacy $(1988)^{38}$ & 4 & $\begin{array}{l}4 \text { T4-6 males, } 20-35 \text { years, } \\
1-8 \text { years after injury }\end{array}$ & Pre-Post trial & $\begin{array}{l}\text { Quadriceps muscle } \\
\text { stimulated: (1) leg } \\
\text { raising against graded } \\
\text { load }-5 \times / \text { week, } \\
10 \text { weeks; } \\
\text { (2) cycling on modified } \\
\text { bicycle ergometer- } \\
32 \text { weeks }\end{array}$ & $\begin{array}{l}\text { BMC by DPA of the } \\
\text { lumbar spine and right } \\
\text { femur }\end{array}$ & $\begin{array}{l}\text { No changes in BMC } \\
\text { or bone density }\end{array}$ \\
\hline
\end{tabular}

$\begin{array}{lll}\begin{array}{l}\text { Kiratli } \\ (1990)^{39}\end{array} & 4 & \begin{array}{l}6 \mathrm{SCl}>2 \text { years }-25 \text { years Pre-Post trial } \\ \text { after injury }\end{array} \\ \text { Leeds } & 4 & \begin{array}{l}6 \mathrm{C} 4-6 \text { males, } 18-27 \text { years, Pre-Post trial } \\ 2-9 \text { years after injury. } 2 \\ \text { paraplegic and } 1 \text { tetraplegic } \\ \text { males, } 24-31 \text { years, trained } \\ \text { for } 3 \text { years }\end{array} \\ & \end{array}$

\begin{tabular}{|c|c|c|}
\hline $\begin{array}{l}\text { Rodgers } \\
(1991)^{41}\end{array}$ & 4 & $\begin{array}{l}12 \text { (3 females) C4-T10 SCI, } \\
5 \mathrm{C} \text { and } 7 \mathrm{IC} \text {; } 19-63 \text { years; } \\
0.7-17 \text { years after injury }\end{array}$ \\
\hline
\end{tabular}

\begin{tabular}{|c|c|c|c|}
\hline $\begin{array}{l}\text { Sloan } \\
(1994)^{42}\end{array}$ & 4 & $\begin{array}{l}\text { C7, IC female, } 40 \text { years, } \\
8 \text { months after injury } \\
\text { C6, IC male, } 55 \text { years, } \\
4.5 \text { years after injury }\end{array}$ & Pre-Post trial \\
\hline $\begin{array}{l}\text { Hangartner } \\
(1994)^{43}\end{array}$ & 4 & $\begin{array}{l}15 \text { C } 5-T 10,17-46 \text { years, } \\
0.3-15.4 \text { years after injury }\end{array}$ & Pre-Post trial \\
\hline
\end{tabular}

\begin{tabular}{|c|c|c|c|}
\hline $\begin{array}{l}\text { BeDell } \\
(1996)^{44}\end{array}$ & 4 & $\begin{array}{l}12 \text { C5-T12, AIS A males, } \\
23-46 \text { years, 2-19 years } \\
\text { after injury }\end{array}$ & Pre-Post trial \\
\hline
\end{tabular}

$\begin{array}{ll}\begin{array}{l}\text { Bloomfield } \\ (1996)^{45}\end{array} & \begin{array}{l}9 \text { C } 5-\text { T7 Frankel A-B, } \\ 21-39 \text { years, 3-12 years } \\ \text { after injury }\end{array}\end{array}$

$\begin{array}{ll}\text { Mohr } & 4 \\ (1997)^{46} & \begin{array}{l}10 \mathrm{C} 6-\mathrm{T} 4 \mathrm{SCl}, 27-45 \\ \text { years, } 2-24 \text { years after } \\ \text { injury }\end{array}\end{array}$

Pre-Post trial
FES to the quadriceps femoris muscle 50$60 \mathrm{~min}, 3$ times/week for 8 months

FES conditioning 1 month. Up to 30 mins FES cycle ergometry, $3 \times /$ week for 6 months Extra three males exercised for 3 years

Quadriceps stimulation for knee extension progressive resistance exercise training up to $3 \times /$ week for 36 sessions

Up to 30 min FES cycling $3 \times /$ week. The female trained for 1 year, the male for 6 months

Three did FES-induced knee extension resistance exercise only; 9 did FES cycling only; 3 did both. They did 1-5 exercise periods of 36 sessions, median of 14.5 weeks

\section{(1) Quadriceps strengthening, (2) up to 30 min FES} cycling, (3a) FES cycling for 30 min, $68 \pm 14$ sessions;

(3b) 8 continued combination of upper and lower extremity FES cycling for $37 \pm 15$ extra sessions

9 months FES cycling: 4 participants trained at $\geqslant 18 \mathrm{~W}$ (Watts) for at least 3 months

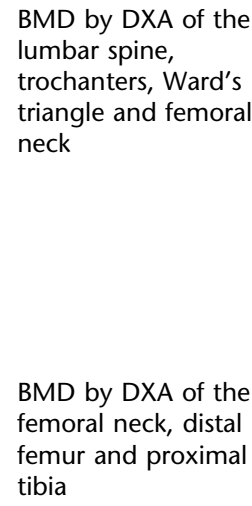

BMD by DXA of the femoral neck, distal femur and proximal tibia

BMD by DXA of the lumbar spine, trochanters, Ward's triangle and femoral neck

BMD method or details not described

No significant change in BMD

BMD by DXA of the No restoration of lumbar spine, trochanter, BMD

and femoral
BMD by special isotope-based CT of tibia
BMD by DPA of the
lumbar spine and

No change proximal femur

BMD by DPA of Ward's No increase in BMD gle, trochanter

femoral neck and

proximal femur 
Table 5 Continued

\begin{tabular}{|c|c|c|c|c|c|c|}
\hline Author (year) & $\begin{array}{l}\text { Level of } \\
\text { evidence }\end{array}$ & Population & Design & Intervention & Measurements & Results \\
\hline $\begin{array}{l}\text { Bélanger } \\
(2000)^{47}\end{array}$ & 2 & $\begin{array}{l}14 \text { ( } 3 \text { females) C5-T5, } \\
\text { Frankel A-C, } 2 \text { IC and } \\
12 \text { C; } 23-42 \text { years; } 1.2-23 \\
\text { years after injury }\end{array}$ & $\begin{array}{l}\text { Controlled } \\
\text { within-subject, } \\
\text { contralateral limb } \\
\text { Pre-Post trial }\end{array}$ & $\begin{array}{l}1 \mathrm{~h} / \text { day, } 5 \text { days/week, } \\
\text { for } 24 \text { weeks: left } \\
\text { quadriceps stimulated } \\
\text { to contraction against } \\
\text { isokinetic load (resisted) } \\
\text { vs right quadriceps } \\
\text { contracted against } \\
\text { gravity (unresisted) }\end{array}$ & $\begin{array}{l}\text { BMD by DXA of the } \\
\text { distal femur, proximal } \\
\text { tibia and mid-tibia }\end{array}$ & $\begin{array}{l}\text { Distal femur and } \\
\text { proximal tibia nearly } \\
30 \% \text { recovery of the } \\
\text { lost bone }(P<0.05) \text {. } \\
\text { No difference } \\
\text { between resisted or } \\
\text { unresisted training }\end{array}$ \\
\hline $\begin{array}{l}\text { Chen } \\
(2005)^{48}\end{array}$ & 4 & $\begin{array}{l}15 \text { C } 5 \text {-T8 complete motor } \\
\text { SCI males, } 23-37 \text { years; } \\
3-16 \text { years after injury }\end{array}$ & Pre-Post trial & $\begin{array}{l}30 \text { min FES cycling } 5 \\
\text { times/day for } 6 \text { months, } \\
\text { followed by } 6 \text { months } \\
\text { without intervention }\end{array}$ & $\begin{array}{l}\text { BMD by DXA of the } \\
\text { lumbar spine, femoral } \\
\text { neck, distal femur, } \\
\text { proximal tibia and } \\
\text { calcaneus }\end{array}$ & $\begin{array}{l}\text { BMD of the distal } \\
\text { femur, proximal tibia } \\
\text { increased }(P<0.05) \text {, } \\
\text { but decreased } \\
\text { significantly without } \\
\text { intervention } \\
(P<0.05)\end{array}$ \\
\hline $\begin{array}{l}\text { Cavalho } \\
(2006)^{49}\end{array}$ & 2 & $\begin{array}{l}21 \text { C4-8 AIS A-B males; } \\
\text { age } 31.95 \pm 8.01 \text { years; } \\
25-180 \text { months after } \\
\text { injury }\end{array}$ & $\begin{array}{l}\text { Controlled } \\
\text { pre-post trial }\end{array}$ & $\begin{array}{l}\text { Gait group: } 11 \text { AIS A } \\
\text { electrically stimulated } \\
\text { treadmill gait with } \\
30-50 \% \text { body weight } \\
\text { support for } 20 \text { min, } 2 \\
\text { times/week for } 6 \text { months } \\
\text { Control group: } 10 \text { AIS B } \\
\text { no gait training }\end{array}$ & $\begin{array}{l}\text { BMD by DXA of the } \\
\text { lumbar spine, proximal } \\
\text { femur and total femur }\end{array}$ & $\begin{array}{l}\text { Differences between } \\
\text { before and after or } \\
\text { between groups not } \\
\text { calculated, but is } \\
\text { insignificant }\end{array}$ \\
\hline $\begin{array}{l}\text { Shields } \\
(2007)^{50}\end{array}$ & 2 & $\begin{array}{l}4 \text { T1-T7 AIS A males; } \\
37-68 \text { years; } 2-12 \text { years } \\
\text { post injury }\end{array}$ & $\begin{array}{l}\text { Pre-Post trial, } \\
\text { within subject, } \\
\text { contralateral } \\
\text { limb control }\end{array}$ & $\begin{array}{l}\text { Trained soleus muscle } \\
\text { using an isometric } \\
\text { plantar flexion electrical } \\
\text { stimulation } 30 \text { min/day, } \\
5 \text { days/week for } 6-11 \\
\text { months. Untrained limb } \\
\text { control }\end{array}$ & $\begin{array}{l}\text { BMD by DXA of } \\
\text { proximal tibia }\end{array}$ & $\begin{array}{l}\text { BMD for proximal } \\
\text { tibia did not differ } \\
\text { before and after } \\
\text { training }\end{array}$ \\
\hline $\begin{array}{l}\text { Frotzler } \\
(2008)^{51}\end{array}$ & 4 & $\begin{array}{l}11 \text { (2 females) traumatic } \\
\text { T3-9 AIS A; } 41.9 \pm 7.5 \\
\text { years; } 11.0 \pm 7.1 \text { years } \\
\text { after injury }\end{array}$ & Pre-Post trial & $\begin{array}{l}\text { Preconditioning until } \\
\text { FES cycling for } 10 \text { min } \\
\text { without resistance. FES } \\
\text { to gluteal, quadriceps } \\
\text { and hamstring muscles. } \\
3 \text { months FES-cycling } \\
\text { for upto } 1 \mathrm{~h} / \text { day. } \\
9 \text { months FES-cycling } \\
\text { for } 1 \mathrm{~h} / \text { day }\end{array}$ & $\begin{array}{l}\text { BMD by PQCT } \\
\text { bilaterally in the femur } \\
\text { and tibia before } \\
\text { conditioning, after } 6 \\
\text { and } 12 \text { months of FES } \\
\text { cycling }\end{array}$ & $\begin{array}{l}\text { After } 12 \text { months, } \\
\text { trabecular and total } \\
\text { BMD and cross } \\
\text { sectional area in the } \\
\text { distal femoral } \\
\text { epiphysis increased } \\
\text { significantly }\end{array}$ \\
\hline
\end{tabular}

Abbreviations: AIS, American Spinal Injury Association (ASIA) Impairment Scale; BMC, bone mineral content; BMD, bone mineral density; C, complete spinal cord lesion; CT, computer tomography; DXA, dual-energy X-ray absorptiometry; FES, functional electrical stimulation; IC, incomplete spinal cord lesion; pQCT, peripheral computed tomography; $\mathrm{SCl}$, spinal cord injury.

measured at the proximal femur only. The study by De Bruin et al. ${ }^{14}$ indicates that early mobilization led to no or insignificant loss of trabecular bone, whereas the immobilized individuals showed a marked decrease when monitored for 25 weeks. In addition, the recent prospective study by Alekna et al. ${ }^{18}$ found that standing, particularly after 2 years, gave significantly higher BMD in legs, pelvis and the total body. The long follow-up carried out in this study is unique. The other studies in Table 1 were of shorter duration and/or less weight-bearing stimulation.

For the chronic phase (Table 2), the picture seems more uniform, with very little evidence for any gain in BMD when the first year after injury has passed. All these studies were of evidence levels 4 and 5 .

The ineffectiveness of shorter term ( $<3$ months), lessaggressive early intervention therapy and the overall ineffec- tiveness of the chronic-phase studies raise the hypothesis that if a weight-bearing intervention is to be considered, it should be more aggressive and should intervene in the early period after injury if a treatment effect is to be found.

\section{The effect of exercise}

The quality of evidence available for evaluation is poor. The most interesting study in this group with only three investigations of level 5 evidence (Table 3) is by Miyahara et al. ${ }^{32}$ They found that the earlier the athlete started sports after injury, the higher the BMD of the legs, body trunk and the entire body. Further, a longer period of athletic career after restarting was significantly related to higher leg BMD. This result is encouraging, and supports the hypothesis of early intervention after injury (suggested in the weight- 
Table 6 The effect of other physical interventions on bone in individuals with SCI

\begin{tabular}{|c|c|c|c|c|c|c|}
\hline $\begin{array}{l}\text { Author } \\
\text { (year) }\end{array}$ & $\begin{array}{l}\text { Level of } \\
\text { evidence }\end{array}$ & Population & Design & Intervention & Measurements & Results \\
\hline $\begin{array}{l}\text { Petrofsky } \\
(1984)^{52}\end{array}$ & 4 & $\begin{array}{l}2 \mathrm{SCl} \text { individuals, no } \\
\text { further description }\end{array}$ & $\begin{array}{l}\text { Before and after } \\
\text { trial. Case studies }\end{array}$ & $\begin{array}{l}\text { Impact vibration (force } \\
\text { of } 3 \mathrm{Gz} \text {, frequency } 15 \mathrm{~Hz} \text { ) } \\
1 \mathrm{~h} \text { for } 3 \text { days/week for } 3 \\
\text { months }\end{array}$ & $\begin{array}{l}\text { CT scans were used to } \\
\text { quantify the density of } \\
\text { leg bones }\end{array}$ & $\begin{array}{l}53.5 \% \text { increase in } \\
\text { BMD of the proximal } \\
\text { tibia }\end{array}$ \\
\hline $\begin{array}{l}\text { Garland } \\
(1999)^{53}\end{array}$ & 2 & $\begin{array}{l}6 \text { complete } \mathrm{SCI} \text { males, } \\
2 \text { tetraplegic and } 4 \\
\text { paraplegic; } 20-39 \text { years; } \\
3-17 \text { years after injury }\end{array}$ & $\begin{array}{l}\text { Repeated- } \\
\text { measures, within- } \\
\text { subject } \\
\text { contralateral limb } \\
\text { control }\end{array}$ & $\begin{array}{l}\text { On one knee } \\
\text { stimulated with pulsed } \\
\text { electromagnetic fields } \\
\text { for minimum of } 8 \mathrm{~h} / \text { day } \\
\text { for } 6 \text { months. The } \\
\text { opposite knee control }\end{array}$ & $\begin{array}{l}\text { BMD by DXA of the } \\
\text { knees initial, after } 3 \text {, } \\
6 \text { and } 12 \text { months }\end{array}$ & $\begin{array}{l}\text { At } 3 \text { months, BMD } \\
\text { increased in the } \\
\text { stimulated knee } \\
(P<0.05) \text { and } \\
\text { declined in the } \\
\text { control knee. By } \\
6 \text { months, BMD } \\
\text { returned to near } \\
\text { baseline values, and } \\
\text { at } 12 \text { months, both } \\
\text { knees had lost bone } \\
\text { at similar rate. Larger } \\
\text { effect closer to the } \\
\text { stimulation }\end{array}$ \\
\hline $\begin{array}{l}\text { Warden } \\
(2001)^{54}\end{array}$ & 1 & $\begin{array}{l}15 \text { C5-T10, AIS A-B, } 17-40 \\
\text { years; } 46-153 \text { days post } \\
\text { injury }\end{array}$ & $\begin{array}{l}\text { Double-blinded, } \\
\text { within-subject } \\
\text { randomized } \\
\text { controlled trial } \\
\text { with contralateral } \\
\text { limb placebo } \\
\text { treated }\end{array}$ & $\begin{array}{l}\text { Low-intensity pulsed } \\
\text { ultrasound on one heel } \\
20 \text { min/day, } 5 \text { days/week } \\
\text { over } 6 \text { weeks. The other } \\
\text { heel placebo treated }\end{array}$ & $\begin{array}{l}\text { Calcaneal BMC by } \\
\text { DXA }\end{array}$ & $\begin{array}{l}\text { No difference } \\
\text { between active and } \\
\text { inactive treatment }\end{array}$ \\
\hline
\end{tabular}

Abbreviations: AIS, American Spinal Injury Association (ASIA) Impairment Scale; BMC, bone mineral content; BMD, bone mineral density; CT, computer tomography; DXA, dual-energy X-ray absorptiometry; SCl, spinal cord injury.

Table 7 The influence of spasticity/spasms on bone in individuals with SCI

\begin{tabular}{|c|c|c|c|c|c|c|}
\hline Author (year) & $\begin{array}{l}\text { Level of } \\
\text { evidence }\end{array}$ & Population & Design & Spasticity & Measurements & Results \\
\hline $\begin{array}{l}\text { Biering- } \\
\text { Sørensen } \\
(1988)^{19}\end{array}$ & 5 & $\begin{array}{l}16 \text { thoracic and lumbar } \mathrm{SCl} \\
\text { for } 2-25 \text { years, acquired at } \\
\text { the age of } \geqslant 16 \text { years }\end{array}$ & $\begin{array}{l}\text { Cross-sectional } \\
\text { Descriptive }\end{array}$ & $\begin{array}{l}6 \text { with spasticity } \\
10 \text { without spasticity }\end{array}$ & $\begin{array}{l}\text { BMC by DPA of the } \\
\text { lumbar spine, femoral } \\
\text { neck and shaft, and } \\
\text { proximal tibia }\end{array}$ & No effect of spasticity \\
\hline
\end{tabular}

\begin{tabular}{|c|c|c|c|c|c|c|}
\hline $\begin{array}{l}\text { Wilmet } \\
(1995)^{55}\end{array}$ & 4 & $\begin{array}{l}31 \text { ( } 7 \text { females) traumatic } \\
\text { T2-L3; mean age } 32.5 \text { years } \\
\text { (17.5-65.5); within } 8 \text { weeks } \\
\text { of injury }\end{array}$ & Prospective & 11 spastic; 12 flaccid & $\begin{array}{l}\text { BMC by DXA of the } \\
\text { regional and total body } \\
10,20,30,40 \text { and } 50 \\
\text { weeks after injury, with } \\
26 \text { of } 2 \text { measurements } \\
\text { at each time point }\end{array}$ & $\begin{array}{l}\text { BMC of the paralysed } \\
\text { areas varies in a very } \\
\text { similar way in the } \\
\text { lower limbs of spastic } \\
\text { and flaccid patients }\end{array}$ \\
\hline $\begin{array}{l}\text { Demirel } \\
(1998)^{56}\end{array}$ & 5 & $\begin{array}{l}41 \text { (9 females) } 5 \text { C } 4-5,6 \\
\text { T1-6, } 20 \text { T7-12, } 10 \text { L1-3; } \\
19-49 \text { years; } 2-30 \text { months } \\
\text { after injury }\end{array}$ & $\begin{array}{l}\text { Cross-sectional } \\
\text { Descriptive }\end{array}$ & $\begin{array}{l}\text { Spasticity assessed on } \\
\text { the Ashworth Peterson } \\
\text { scale }(0-4) ; 35 \text { were } \\
\text { spastic: } 13, \text { grade } 1 ; \\
14 \text {, grade } 2 ; 4, \text { grade } 3 ; \\
4, \text { grade } 4 ; \text { and } 6 \text {, had } \\
\text { no spasticity (numbers } \\
\text { and percentages do } \\
\text { not match) }\end{array}$ & $\begin{array}{l}\text { BMD by DXA of the } \\
\text { whole body using arms } \\
\text { and legs }\end{array}$ & $\begin{array}{l}\text { Those with spasticity } \\
\text { had higher BMD } \\
\text { when compared with } \\
\text { flaccid individuals } \\
(P<0.05)\end{array}$ \\
\hline $\begin{array}{l}\text { Frey-Rindova } \\
(2000)^{15}\end{array}$ & 4 & $\begin{array}{l}24 \text { males, traumatic } \mathrm{SCl} \\
\text { C4-L1 Frankel A-C } \\
\text { completed the study }\end{array}$ & $\begin{array}{l}\text { Prospective } \\
\text { repeated } \\
\text { measures }\end{array}$ & $\begin{array}{l}\text { Spasticity assessed on } \\
\text { the Ashworth Peterson } \\
\text { scale (0-4). 5: grade } 1 ; \\
11: \text { grade } 2 ; 8 \text { : grade } 3\end{array}$ & $\begin{array}{l}\text { BMD by } \mathrm{pQCT} \text { of the } \\
\text { tibia } 1,6,12 \text { months } \\
\text { after } \mathrm{SCl}\end{array}$ & $\begin{array}{l}\text { Spasticity assessed by } \\
\text { the Ashworth scale } \\
\text { did not significantly } \\
\text { influence the BMD } \\
\text { loss }\end{array}$ \\
\hline
\end{tabular}


Table 7 Continued

\begin{tabular}{|c|c|c|c|c|c|c|}
\hline Author (year) & $\begin{array}{l}\text { Level of } \\
\text { evidence }\end{array}$ & Population & Design & Spasticity & Measurements & Results \\
\hline Eser $(2005)^{28}$ & 5 & $\begin{array}{l}54 \text { ( } 5 \text { females) traumatic } \\
\text { C5-L3 AIS A and B; } 24-72 \\
\text { years; } 5-48 \text { years after } \\
\text { injury; } 6 \text { acquired SCI } \\
\text { before } 18 \text { years }\end{array}$ & $\begin{array}{l}\text { Cross-sectional } \\
\text { Descriptive }\end{array}$ & $\begin{array}{l}\text { Spasticity assessed with } \\
\text { the Ashworth scale for } \\
\text { knee flexion/extension, } \\
\text { hip abduction/ } \\
\text { adduction/flexion/ } \\
\text { extension summed for } \\
\text { each leg. } 48 \text { spastic; } \\
6 \text { flaccid }\end{array}$ & $\begin{array}{l}\text { BMD by } \mathrm{PQCT} \text { of the } \\
\text { radius, femur and tibia }\end{array}$ & $\begin{array}{l}\text { Spasticity } \\
\text { measurement vs } \\
\text { pQCT of femur: total } \\
\text { BMD }(P=0.008) \text {, } \\
\text { trabecular BMD of } \\
\text { distal epiphysis } \\
(P=0.014) \text {, and the } \\
\text { cortical thickness of } \\
\text { shaft }(P=0.003) \text {. } \\
\text { Spasticity was not } \\
\text { found to increase } \\
\text { bone mass of the } \\
\text { lower leg }\end{array}$ \\
\hline $\begin{array}{l}\text { Shojaei } \\
(2006)^{57}\end{array}$ & 5 & $\begin{array}{l}132 \text { male veterans with } \\
\text { complete paralysis; } 12.9 \% \\
\text { cervical, } 78 \% \text { thoracic, } 9.1 \% \\
\text { lumbar; } 25-51 \text { years; } 5-23 \\
\text { years after injury }\end{array}$ & $\begin{array}{l}\text { Cross-sectional } \\
\text { Descriptive }\end{array}$ & $\begin{array}{l}37.1 \% \text { severe spasticity } \\
40.1 \% \text { had mild-to- } \\
\text { moderate spasticity } \\
22.7 \% \text { had no } \\
\text { experience of spasticity } \\
\text { Assessment method not } \\
\text { specified }\end{array}$ & $\begin{array}{l}\text { BMD by DXA of the L4 } \\
\text { and neck of right femur }\end{array}$ & $\begin{array}{l}\text { No statistically } \\
\text { significant relation } \\
\text { between BMD and } \\
\text { spasticity }\end{array}$ \\
\hline
\end{tabular}

Abbreviations: AIS, American Spinal Injury Association (ASIA) Impairment Scale; BMC, bone mineral content; BMD, bone mineral density; DPA, dual-energy photon absorptiometry; DXA dual-energy X-ray absorptiometry; $\mathrm{PQCT}$, peripheral computed tomography; SCl, spinal cord injury.

bearing analysis) if a treatment effect is to be found in future studies.

\section{The effect of electrical stimulation}

Of the four level 2 studies performed early after SCI (Table 4), three showed that there may be some effect of electrical stimulation with stimulation of 5 days/week. The single-case long-time prospective investigation by Dudley-Javoroski and Shields ${ }^{37}$ additionally suggests the importance of where the muscles stress the bone. This further emphasizes the value of specific methods for BMD measurement, that is in most studies, the dual-energy X-ray absorptiometry ${ }^{35,36,42,44-50}$ is used as it is considered to be the 'gold standard,' but peripheral quantitative computed tomography has the advantage of being able to differentiate the cortical from the trabecular bone, and assess both bone geometry and volumetric density. ${ }^{14,15,37,51}$ A quantitative ultrasound has also been used, but probably is still not suitable to monitor the effect of intervention on $\mathrm{BMD},{ }^{58}$ whereas magnetic resonance imaging also seems to have a potential in future studies in assessing bone geometry. ${ }^{59}$

For the chronic phase (Table 5), the studies are conflicting, but those investigations that show improvement seem to be those with a longer period of training, that is 12 months, ${ }^{46}$ or higher frequency, that is 5 times/week $^{47,48}$ or stimulus intensity. ${ }^{45}$ It is also evident that all these studies measured their improvement corresponding to the trabecular bone, in particular in the distal femur or the proximal tibia. The challenge may be to provide sufficient mechanical stimulus without increasing the risk of fracture. $^{12,60}$
It is also observed that the positive effect of electrical stimulation on the bone mass only remains if the stimulation is continued and in sufficient amounts. ${ }^{46,48}$ Therefore, the recommendation following the experience gained from the studies is that the electrical stimulation should be at least 2-3 times per week, and probably has to be continued for the long term if the bone mass is not to decline further.

\section{The effect of other non-pharmacological interventions}

Table 6 shows that the only randomized study by Warden et al. ${ }^{54}$ did not indicate any benefit from low-intensity pulsed ultrasound. Potentially, both impact vibration ${ }^{52}$ and pulsed electromagnetic fields ${ }^{53}$ showed positive results, but the studies are of poor quality. These results could support the hypothesis that a more dynamic loading may be necessary to elicit a detectable treatment effect.

\section{The influence of spasticity/spasms}

The studies evaluating the possible influence of spasticity (Table 7) show inconsistent results. Demirel et al. ${ }^{56}$ found that those with spasticity had higher BMD when compared with flaccid individuals, and Eser et al. ${ }^{28}$ showed a significant correlation between the degree of spasticity measured with the modified Ashworth scale and BMD.

The available studies are generally of a low level of evidence, and do not support the hypothesis that spasticity maintains bone mineral in individuals with SCI.

\section{Concluding remarks}

The level of evidence is important and in this study area, there is no conclusive indication of any effective interven- 
tion. This review illustrates that there are issues important in the design of future clinical trials in this area. This includes where the BMD is measured (which is most relevant to be performed around the knee), and the length and intensity of the non-pharmacological intervention carried out. It is important to be aware that the stimulation used has to be maintained if the positive effect on BMD is to remain.

It has to be acknowledged that longitudinal randomized controlled investigations over very long periods on osteoporosis in individuals with SCI are difficult to carry through because of the relatively small number of persons with SCI available for the studies and a potentially high drop out rate over a long follow-up period. At the same time, adequate matching by gender, age, level and completeness of lesion, and time after injury is a challenge to the potential number of individuals recruited. ${ }^{4}$ Unfortunately, if the hypothesis regarding aggressive, high-intensity therapies is true, the ability to practically design and perform within patient evaluations to reduce sample size requirements as conducted by Ben et al. ${ }^{16}$ and Warden et al. ${ }^{54}$ becomes more challenging.

The detection of an effective clinical intervention in this study area will require rigorous minimization of bias through a randomized controlled clinical trial design and likely require the involvement of multiple centers. It will also be dependent on appropriate measurement, with adequate intensity and duration of the intervention to detect a treatment effect. Any prospective intervention is likely to benefit from early timing after acute SCI.

\section{References}

1 Biering-Sørensen F, Bohr HH, Schaadt OP. Longitudinal study of bone mineral content in the lumbar spine, the forearm and the lower extremities after spinal cord injury. Eur J Clin Invest 1990; 20: 330-335.

2 Garland DE, Stewart CA, Adkins RH, Hu SS, Rosen C, Liotta FJ et al. Osteoporosis after spinal cord injury. J Orthop Res 1992; 10: 371-378.

3 Jiang S-D, Dai L-Y, Jiang L-S. Osteoporosis after spinal cord injury. Osteoporos Int 2006; 17: 180-192.

4 Giangregorio L, McCartney N. Bone loss and muscle atrophy in spinal cord injury: epidemiology, fracture prediction, and rehabilitation strategies. J Spinal Cord Med 2006; 29: 489-500.

5 Garland DE, Adkins RH, Stewart CA, Ashford R, Vigil D. Regional osteoporosis in women who have a complete spinal cord injury. J Bone Joint Surg (Am) 2001; 83: 1195-1200.

6 Garland DE, Adkins RH, Kushwaha V, Stewart CA. Risk factors for osteoporosis at the knee in the spinal cord injury population. J Spinal Cord Med 2004; 27(Suppl 3): 202-206.

7 Vestergaard P, Krogh K, Rejnmark L, Mosekilde L. Fracture rates and risk factors for fractures in patients with spinal cord injury. Spinal Cord 1998; 36: 790-796.

8 Logan Jr WC, Sloane R, Lyles KW, Goldstein B, Hoenig HM. Incidence of fractures in a cohort of veterans with chronic multiple sclerosis or traumatic spinal cord injury. Arch Phys Med Rehabil 2008; 89: 237-243.

9 Ashe MC, Craven BC, Eng JJ, Krassioukov A, the SCIRE Research Team. Prevention and treatment of bone loss after a spinal cord injury: a systematic review. Topics Spinal Cord Inj Rehabil 2007; 13: 123-145.

10 Ashe MC, Craven C, Krassioukov A, Eng JJ. Bone health following spinal cord injury. In: Eng JJ, Teasell RW, Miller WC, Wolfe DL, Townson AF, Aubut J, Abramson C, Hsieh JTC, Connolly S (eds). Spinal Cord Injury Rehabilitation Evidence. Vancouver, 2006; pp 9.1-9.18; available at http://www.icord.org/scire.
11 Giangregorio LM, Webber CE, Phillips SM, Hicks AL, Craven BC, Bugaresti JM et al. Can body weight supported treadmill training increase bone mass and reverse muscle atrophy in individuals with chronic incomplete spinal cord injury? Appl Physiol Nutr Metab 2006; 31: 283-291.

12 Maimoun L, Fattal C, Micallef J-P, Peruchon E, Rabischong P. Bone loss in spinal cord-injured patients: from physiopathology to therapy. Spinal Cord 2006; 44: 203-210.

13 Methods of the systematic reviews. In: Eng JJ, Teasell RW, Miller WC, Wolfe DL, Townson AF, Aubut J, Abramson C, Hsieh JTC, Connolly S (eds). Spinal Cord Rehabilitation Evidence 2006. Vancouver, pp 2.1-2.11. Available at http://www.icord.org/scire/ pdf/SCIRE_CH2.pdf.

14 De Bruin ED, Frey-Rindova P, Herzog RE, Dietz V, Dambacher MA, Strüssi E. Changes of tibia bone properties after spinal cord injury: effects of early intervention. Arch Phys Med Rehabil 1999; 80: 214-220.

15 Frey-Rindova P, de Bruin ED, Strüssi E, Dambacher MA, Dietz V. Bone mineral density in upper and lower extremities during 12 months after spinal cord injury measured by peripheral quantitative computed tomography. Spinal Cord 2000; 38: 26-32.

16 Ben M, Harvey L, Denis S, Glinsky J, Goehl G, Chee S et al. Does 12 weeks of regular standing prevent loss of ankle mobility and bone mineral density in people with recent spinal cord injuries? Aust J Physiother 2005; 51: 251-256.

17 Giangregorio LM, Hicks AL, Webber CE, Phillips SM, Craven BC, Bugaresti JM et al. Body weight supported treadmill training in acute spinal cord injury: impact on muscle and bone. Spinal Cord 2005; 43: 649-657.

18 Alekna V, Tamulaitiene M, Sinevicius T, Juocevicius A. Effect of weight-bearing activities on bone mineral density in spinal cord injured patients during the period of the first two years. Spinal Cord 2008; 46: 727-732.

19 Biering-Sørensen F, Bohr H, Schaadt O. Bone mineral content of the lumbar spine and lower extremities years after spinal cord lesion. Paraplegia 1988; 26: 293-301.

20 Saltzstein RJ, Hardin S, Hastings J. Osteoporosis in spinal cord injury: using an index of mobility and its relationship to bone density. J Am Paraplegia Soc 1992; 15: 232-234.

21 Kunkel CF, Scremin AME, Eisenberg B, Garcia JF, Roberts S, Martinez S. Effect of 'standing' on spasticity, contracture, and osteoporosis in paralyzed males. Arch Phys Med Rehabil 1993; 74: 73-78.

22 Ogilvie C, Bowker P, Rowley DI. The physiological benefits of paraplegic orthotically aided walking. Paraplegia 1993; 31: 111-115.

23 Goemaere S, van Laere M, de Neve P, Kaufman JM. Bone mineral status in paraplegic patients who do or do not perform standing. Osteoporosis Int 1994; 4: 138-143.

24 Thoumie P, leClaire G, Beillot J, Dassonville J, Chevalier T, Perrouin-Verbe B et al. Restoration of functional gait in paraplegic patients with the RGO-II hybrid orthosis. A multicenter controlled study. II: physiological evaluation. Paraplegia 1995; 33: 654-659.

25 Needham-Shropshire BM, Broton JG, Klose KJ, Lebwohl N, Guest RS, Jacobs PL. Evaluation of a training program for persons with SCI paraplegia using Parastep $₫ 1$ ambulation system: Part 3. Lack of effect on bone mineral density. Arch Phys Med Rehabil 1997; 78: 799-803.

26 Dauty M, Verbe BP, Maugars Y, Dubois C, Mathe JF. Supralesional and sublesional bone mineral density in spinal cord-injured patients. Bone 2000; 27: 305-309.

27 Sabo D, Blaich S, Wenz W, Hohmann M, Loew M, Gerner HJ. Osteoporosis in patients with paralysis after spinal cord injury. A cross sectional study in 46 male patients with dual-energy X-ray absorptiometry. Arch Orthop Trauma Surg 2001; 121: 75-78.

28 Eser P, Frotzler A, Zehnder Y, Schiessl H, Denoth J. Assessment of anthropometric, systemic, and lifestyle factors influencing bone status in the legs of spinal cord injured individuals. Osteoporos Int 2005; 16: 26-34.

29 Goktepe AS, Tugcu I, Yilmaz B, Alaca R, Gunduz S. Does standing protect bone density in patients with spinal cord injury? J Spinal Cord Med 2008; 31: 197-201. 
30 Jones LM, Legge M, Goulding A. Intensive exercise may preserve bone mass of the upper limbs in spinal cord injured males but does not retard demineralisation of the lower body. Spinal Cord 2002; 40: 230-235.

31 Goktepe AS, Yilmaz B, Alaca R, Yazicioglu K, Mohur H, Gunduz S. Bone density loss after spinal cord injury. Elite paraplegic basketball players vs. paraplegic sedentary persons. Am J Phys Med Rehabil 2004; 83: 279-283.

32 Miyahara K, Wang D-H, Mori K, Takahashi K, Miyatake N, Wang B-L et al. Effect of sports activity on bone mineral density in wheelchair athletes. J Bone Moner Metab 2008; 26: 101-106.

33 Eser P, de Bruin ED, Telley I, Lechner HE, Knecht H, Strüssi E. Effect of electrical stimulation-induced cycling on bone mineral density in spinal cord-injured patients. Eur J Clin Invest 2003; 33: 412-419.

34 Shields RK, Dudley-Javoroski S. Musculoskeletal plasticity after acute spinal cord injury: effects of long-term neuromuscular electrical stimulation training. I Neurophysiol 2006; 95: 2380-2390.

35 Shields RK, Dudley-Javoroski S, Law LAF. Electrical induced muscle contractions influence bone decline after spinal cord injury. Spine 2006; 31: 548-553.

36 Clark JM, Jelbart M, Rischbieth H, Strayer J, Chatterton B, Schultz C et al. Physiological effects of lower extremity functional electrical stimulation in early spinal cord injury: lack of efficacy to prevent bone loss. Spinal Cord 2007; 45: 78-85.

37 Dudley-Javoroski S, Shields RK. Dose estimation and surveillance of mechanical loading interventions for bone loss after spinal cord injury. Phys Ther 2008; 88: 387-396.

38 Pacy PJ, Hesp R, Halliday DA, Katz D, Cameron G, Reeve J. Muscle and bone in paraplegic patients, and the effect of functional electrical stimulation. Clin Sci 1988; 75: 481-487.

39 Kiratli BJ, Agre JC. Disuse osteoporosis in spinal cord injured patients. J Rehabil Res Develop 1991; 28: 425-426.

40 Leeds EM, Klose KJ, Ganz W, Serafini A, Green BA. Bone mineral density after bicycle ergometry training. Arch Phys Med Rehabil 1990; 71: 207-209.

41 Rodgers MM, Glaser RM, Figoni SF, Hooker SP, Ezenwa BN, Collins SR et al. Musculoskeletal responses of spinal cord injured individuals to functional neuromuscular stimulation-induced knee extension exercise training. I Rehabil Res Dev 1991; 28: $19-26$.

42 Sloan KE, Bremner LA, Byrne J, Day RE, Scull ER. Musculoskeletal effects of an electrical stimulation induced cycling programme in the spinal injured. Paraplegia 1994; 32: 407-415.

43 Hangartner TN, Rodgers MM, Glaser RM, Barre PS. Tibial bone density loss in spinal cord injured patients: effects of FES exercise. J Rehabil Res Devel 1994; 31: 50-61.

44 BeDell KK, Scremin AME, Perell KL, Kunkel CF. Effects of functional electrical stimulation-induced lower extremity cycling on bone density of spinal cord-injured patients. Am J Phys Med 1996; 75: 29-34.

45 Bloomfield SA, Mysiw WJ, Jackson RD. Bone mass and endocrine adaptations to training in spinal cord injured individuals. Bone 1996; 19: 61-68.
46 Mohr T, Pødenphant J, Biering-Sørensen F, Galbo H, Tramsborg G, Kjær M. Increased bone mineral density after prolonged electrical induced cycle training of paralyzed limbs in spinal cord injured man. Calcif Tissue Int 1997; 61: 22-25.

47 Bélanger M, Stein RB, Wheeler GD, Gordon T, Leduc B. Electrical stimulation: can it increase muscle strength and reserve osteopenia in spinal cord injured individuals? Arch Phys Med Rehabil 2000; 81: 1090-1098.

48 Chen S-C, Lai C-H, Chan WP, Huang M-H, Tsai H-W, Chen J-JJ. Increases in bone mineral density after functional electrical stimulation cycling exercises in spinal cord injured patients. Disabil Rehabil 2005; 27: 1337-1341.

49 Cavalho DCL, Garlipp CR, Bottini PV, Afaz SH, Moda MA, Cliquet $\mathrm{Jr}$ A. Effect of treadmill gait on bone markers and bone mineral density of quadriplegic subjects. Braz J Med Biol Res 2006; 39: 1357-1363.

50 Shields RK, Dudley-Javoroski S. Musculoskeletal adaptations in chronic spinal cord injury: effects of long-term soleus electrical stimulation training. Neurorehabil Neural Repair 2007; 21: 169-179.

51 Frotzler A, Coupaud S, Perret C, Kakebeeke TH, Hunt KJ, Donaldson NdN et al. High-volume FES-cycling partially reverses bone loss in people with chronic spinal cord injury. Bone 2008; 43: $169-176$.

52 Petrofsky JS, Phillips CA. The use of functional electrical stimulation for rehabilitation of spinal cord injured patients. Cent Nerv Syst Trauma 1984; 1: 57-74.

53 Garland DE, Adkins RH, Matsuno NN, Stewart CA. The effect of pulsed electromagnetic fields on osteoporosis at the knee in individuals with spinal cord injury. J Spinal Cord Med 1999; 22: 239-245.

54 Warden SJ, Bennell KL, Matthews B, Brown DJ, McMeeken JM, Wark JD. Efficacy of low-intensity pulsed ultrasound in the prevention of osteoporosis following spinal cord injury. Bone 2001; 29: 431-436.

55 Wilmet E, Ismail AA, Heilporn A, Welraeds D, Bergmann P. Longitudinal study of the bone mineral content and of soft tissue composition after spinal cord section. Paraplegia 1995; 33: 674-677.

56 Demirel G, Yilmaz H, Paker N, Onel S. Osteoporosis after spinal cord injury. Spinal Cord 1998; 36: 822-825.

57 Shojaei H, Soroush MR, Modirian E. Spinal cord injury-induced osteoporosis in veterans. J Spinal Disord Tech 2006; 19: 114-117.

58 Lewiecki EM, Richmond B, Miller PD. Uses and misuses of quantitative ultrasonography in managing osteoporosis. Cleve Clin J Med 2006; 73: 742-746; 749-752.

59 Liu D, Manske SL, Kontulainen SA, Tang C, Guy P, Oxland TR et al. Tibial geometry is associated with failure load ex vivo: a MRI, pQCT and DXA study. Osteoporos Int 2007; 18: 991-997.

60 Hartkopp A, Murphy RJL, Mohr T, Kjær M, Biering-Sørensen F. Bone fracture during electrical stimulation of the quadriceps in a spinal cord injured subject. Arch Phys Med Rehabil 1998; 79: 1133-1136. 